10. Miner MM. Primary care physician versus urologist: how does their medical management of LUTS associated with BPH differ? Curr Urol Rep 2009;10:254-60.

11. Stafinski T, Menon D, Harris K, et al. Photoselective vaporization of the prostate for the treatment of benign prostatic hyperplasia. Can Urol Assoc J 2008;2:124-34.

12. Elliott SP, Sweet RM. Contemporary surgical management of benign prostatic hyperplasia: what do recent trends imply for urology training? Curr Prostate Rep 2009;7:19-24.
13. Quinlan $M R, O^{\prime}$ Daly $B J, O^{\prime}$ Brien $M F$, et al. The value of appropriate assessment prior to specialist referral in men with prostatic symptoms. Ir J Med Sci 2009;178:281-5.

Correspondence: Dr. Fred Saad, Director of Urologic Oncology, Centre Hospitalier de l'Université de Montréal, CHUM - Pavillon Notre-Dame, 1560 Sherbrooke East, Department of Urology, Montréal, QC H2L 4M1; fax: 514-412-7620; fredsad@ @videotron.ca

\title{
Do more BPH patients mean more urologists or just better management strategies?
}

\author{
J. Curtis Nickel, MD, FRCSC
}

See related article on page 123.

Can Urol Assoc J 2010;4(2):127-8

W e are told that we need more urologists over the next 8 years to manage the increasing number of aging men with benign prostatic hyperplasia (BPH) in Canada over the same period. ${ }^{1}$ There is certainly no doubt that we are seeing a rapidly aging population of men in Canada and that we are going to have to manage all kinds of urology problems associated with this aging population. But do we really need more urologists to manage BPH in Canada? I don't think so!

To start with, can we really absorb the increase in urology manpower in our present system, as suggested by the authors? Not a chance. Based on previous needs assessment of urology manpower, urology training programs dramatically expanded over the last decade. Is this helping? Not really, since many (some would say most) graduating residents are not getting jobs out of residency; they are applying for fellowships to put in time or try to make themselves marketable or they are giving up and going to the United States. This paradox (urology shortage despite an overabundance of graduating trainees looking for employment) is caused by a number of factors:
1. Urologists are not retiring. It may be because of the recent economic downturn or the fact that we do not have a culture of retiring in our profession.

2. Urologists are working harder to make ends meet and, therefore, in our piecework payment system we are either actively discouraging competition or are sucking out the lifeblood of any new urologist trying to set up a practice in our area.

3. The major limitation in increasing manpower is resource restriction. The key to increasing urology manpower is not training hundreds more residents, but providing the residents who do graduate with the local hospital resources to carry out a successful urology practice in Canada.

Increasing urology manpower for our aging population is a great idea, but do we really need to use the management of BPH as the primary argument? There is no reason why BPH cannot be diagnosed and managed by family physicians (FPs). Cases suspicious for prostate cancer (prostatespecific antigen [PSA] levels, abnormal digital rectal examinations [DRE]), refractory to first-line medical therapy, and/or require surgical management because of failure of therapy, choice or progression events and/or complications can be referred to urologists.

Let's take another look at the data the authors have shown us. For my province of Ontario, the authors consider an increase of 103 additional urologists will be necessary by 
2018 just to deal with BPH alone. Is it really practical, possible or even necessary to increase urology manpower by that much to manage the increasing number of elderly men specifically for BPH? I don't think so, and even if I did, it will not happen, not even in our dreams. So what do we do about his potential problem?

To start with, we need to change our approach to $\mathrm{BPH}$. The model I see used by many urologists is one of yearly (or in some cases more frequent) "prostate" follow-up, which usually includes an evaluation of lower urinary tract symptoms (LUTS), review of BPH therapy, a PSA and a DRE. While very remunerative for the urologist, this strategy is entirely unnecessary and a real waste of urological expertise. A more efficient and arguably a much better way to manage BPH patients is the shared-care approach with FPs. In this scenario, the urologist confirms the diagnosis, rules out prostate cancer as best we can, initiates therapy (either watchful waiting, medical or surgical) and then transfers the patient back to the FP. The FP's task is to follow LUTS (no matter what therapy was initiated), monitor for progression and/or complications, monitor PSA (and DRE) if indicated and then refer patients back to the urologist, as necessary. In this algorithm, the expertise of both the urologist and FP is maximized and the urology manpower shortage with respect to $\mathrm{BPH}$ envisioned by the authors will not occur.
Since we are never going to attain the manpower levels desired by the authors, let's use the arguments outlined in their article to better employ realistic projections of new urology manpower that we are churning out of our university programs and increase the necessary resources to keep them in Canada.

Professor of Urology, Queen's University, CIHR Tier One Canada Research Chair in Urology, Kingston, ON

Competing interests: None declared.

This paper has been peer-reviewed.

\section{Reference}

1. Rawson NSB, Saad F. The aging male population and medical care for benign prostatic hyperplasia in Canada. Can Urol Assoc J 2010;4:123-7.

Correspondence: Dr. J. Curtis Nickel, Department of Urology, Queen's University, Kingston General Hospital, Kingston, 0N K7L 2V7; fax: 613-545-1970; jin@queensu.ca 